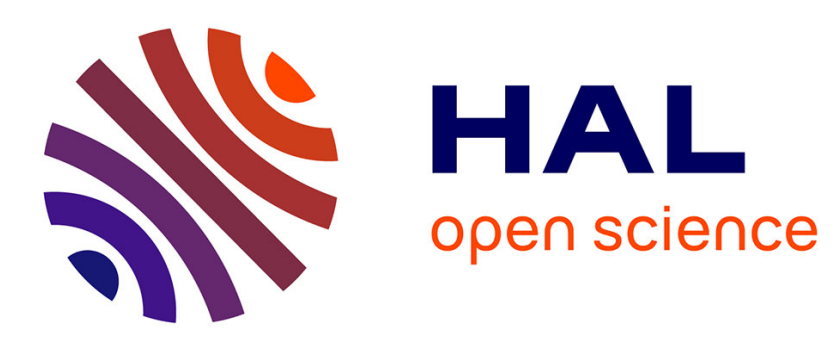

\title{
Perversion narcissique et violence morale en temps de confinement. Note de recherche.
}

\author{
Marc Joly
}

\section{To cite this version:}

Marc Joly. Perversion narcissique et violence morale en temps de confinement. Note de recherche.. Savoir / Agir , 2021, N 55 (1), pp.111-120. 10.3917/sava.055.0111 . hal-03328172

\section{HAL Id: hal-03328172}

\section{https://hal.science/hal-03328172}

Submitted on 28 Aug 2021

HAL is a multi-disciplinary open access archive for the deposit and dissemination of scientific research documents, whether they are published or not. The documents may come from teaching and research institutions in France or abroad, or from public or private research centers.
L'archive ouverte pluridisciplinaire HAL, est destinée au dépôt et à la diffusion de documents scientifiques de niveau recherche, publiés ou non, émanant des établissements d'enseignement et de recherche français ou étrangers, des laboratoires publics ou privés. 


\section{Perversion narcissique et violence morale en temps de confinement. Note de recherche.}

par Marc Joly

Dans le cadre d'une vaste enquête sur la genèse et les usages sociaux de la catégorie de perversion narcissique, entamée en 2017, j'effectuais un travail de terrain depuis plus de six mois dans une association de lutte contre la violence morale domestique quand la propagation de la pandémie de Covid-19 entraîna, en France comme ailleurs, l'adoption de mesures de confinement s'appliquant sur tout le territoire. Le 17 mars 2020, l'association ferma ses portes et ses permanences téléphoniques. Il resta possible de prendre contact avec elle par voie électronique et de demander à être rappelé en laissant un numéro de téléphone. Les psychologues - trois femmes et un homme - maintinrent leurs accompagnements à distance (Skype et téléphone). La présidente et fondatrice de l'association (je la nommerai par la suite Fabienne), spécialisée dans la stratégie juridique et le positionnement, se dota d'emblée d'un nouveau numéro spécial pour être disponible à tout moment auprès des membres ayant besoin de conseils en urgence. La gestionnaire administrative, seule salariée à temps plein de l'association, fut mise en chômage partiel. Enfin, le stage de six étudiantes en psychologie (recrutées dans le cadre de partenariats avec des universités et des établissements privés d'enseignement supérieur) prit fin prématurément.

Le fonctionnement de l'association a donc été fortement perturbé par le confinement. Au début de l'année 2021, il commençait tout juste à reprendre son cours habituel. Une réorganisation administrative a par ailleurs été mise en œuvre.

L'aspect peut-être le plus remarquable des activités que j'avais pu observer pendant six mois réside dans une gestion du temps qui oscille entre l'urgence et la lente décantation. D'un côté, les stagiaires sont invitées à ne pas faire durer les premiers appels au-delà de quinze à vingt minutes ; et c'est toujours le branle-bas de combat, à propos de tel ou tel dossier et surtout quand le sort d'un enfant est en jeu, la veille ou à quelques heures d'un rendez-vous crucial avec une juge, un expert, une assistante sociale ou un éducateur spécialisé. De l'autre côté, les psys et Fabienne ont l'habitude d'échanger longuement sur leurs cas respectifs (en intégrant parfois les stagiaires sur un mode parfaitement symétrique); les documents produits dans le cadre de l'association sont le fruit d'une réflexion collective extrêmement approfondie (ce qui contraste avec le caractère expéditif de certains rapports d'expertise); et les entretiens d'adhésion peuvent durer jusqu'à quatre heures.

Après chaque premier appel, les stagiaires notent sur une fiche la date et la durée de la conversation téléphonique, quelques informations sur la personne appelante ainsi que les premiers éléments de sa demande (par exemple: "veut RDV car relation avec PN [pervers narcissique] » ou «harcèlement ex conjoint/Menace. Parano? a peur »). En bas de cette fiche, elles indiquent enfin la nature du traitement de l'appel : pour l'essentiel, soit un simple mail d'information sera envoyé, soit un rendez-vous d'adhésion sera fixé. Au cours d'un tel rendez-vous, la personne en demande d'aide est invitée à exposer sa problématique. Fabienne 
ou l'une ou l'autre des psychologues présente ensuite l'association et les types d'accompagnement proposés: soutien psychologique, stratégie juridique, aide au positionnement (rédaction de messages électroniques par exemple), tutorats plus spécifiques (séances d'EFT ou d'EMDR ${ }^{1}$ ), etc. Cet échange, intense et poussé, décidera de l'adhésion : devenir membre de l'association, c'est pouvoir bénéficier d'un accompagnement régulier gratuit et de tarifs préférentiels pour tel ou tel tutorat.

Du fait de la situation sanitaire, il ne m'est pour le moment plus possible d'observer de manière continue ces différentes pratiques, dont la raison d'être est de parvenir à distinguer clairement les situations de violence (morale ou psychologique) des situations de conflit : à la faveur d'un diagnostic différentiel, c'est toute la réalité des relations asymétriques, incompréhensible de prime abord, qu'il s'agit de reconnaître par rapport à la réalité des conflits (relations symétriques). Mais il se pourrait bien que ces circonstances exceptionnelles aient créé un observatoire favorisant une meilleure compréhension des phénomènes considérés. C'est ce que je vais tâcher de montrer dans cette note, d'abord en explicitant le cadre théorique que j'essaye de construire, ensuite en présentant les premiers résultats d'une « enquête dans l'enquête » menée « en distanciel».

\section{Quelle sociologie de la violence psychologique?}

La violence d'ordre psychique est un phénomène difficile à appréhender. Présente dans toute forme de violence (physique, sexuelle), elle peut revêtir en même temps une autonomie, une sorte d'autosuffisance qui réside peut-être précisément, du côté de l'auteur ou de l'autrice, dans la jouissance de ne pas exercer d'agressions visibles - de ne pas passer à l'acte dans le sens traditionnel de l'expression. Par violence psychologique ou morale, il faut entendre ainsi un travail de sape qui procède d'une incapacité massive à considérer l'intégrité psychique d'autrui et qui procède par la dénégation et la destruction de toutes les qualités d'un objetcible. Ce n'est pas un hasard si l'inventeur de la notion de perversion narcissique, le psychiatre et psychanalyste Paul-Claude Racamier (1924-1996), a établi une gradation des dénis (du déni psychotique qui efface la réalité même de l'objet au déni qui « ampute l'objet, ainsi que la pensée, de ses propriétés essentielles $\left.{ }^{2} \gg\right)$.

L'association dont il est question n'est pas à proprement parler féministe : elle ne procède pas du mouvement féministe et des victimes hommes y sont accueillies. Il reste que la grande majorité des dossiers concerne des femmes en instance de divorce ou engagées dans des batailles épuisantes à propos de la garde et de l'éducation des enfants, dont l'intégrité psychique est souvent menacée par le parent violent. Pour un sociologue, c'est tout sauf anodin. Entre autres questions, il ne peut éviter de s'interroger sur les manières de s'adapter à la qualification comme «violents » de comportements masculins autrefois normaux et légitimes. Il est probable que le problème ne se pose pas dans les mêmes termes pour les hommes et pour les femmes. Par exemple, exiger de l'épouse qu'elle ne travaille pas était admissible et juridiquement justifié jusqu'en 1965, en France; aujourd'hui, c'est de la «violence conjugale économique »; de même, le contrôle marital de la correspondance privée et des relations de cette même épouse, aboli dix ans plus tard, est désormais ressenti et dénoncé par les pouvoirs publics - comme de l'ordre de l'emprise, de la «violence psychologique », éventuellement de la « cyber-violence ».

La théorie des processus civilisateurs de Norbert Elias est ici incontournable : quand certains comportements autrefois permis sont interdits, quand, parallèlement ou réciproquement, certains comportements proscrits deviennent autorisés, quand, enfin, de nouveaux 
comportements apparaissent dans l'entrelacs de ces mouvements pendulaires, c'est le signe que la structure des rapports sociaux (rapports entre sexes, rapports entre générations, rapports entre groupes) ainsi que l'équilibre des balances du pouvoir afférentes se transforment, et que les normes de sensibilité et schèmes de perception s'ajustent corrélativement ${ }^{3}$.

Comme pour toute configuration générale de changements interconnectés, il importe de choisir un angle d'analyse qui favorise la formulation de questions de recherche précises sans faire perdre de vue l'ensemble des processus configurationnels à l'œuvre. Ainsi, la contrainte sociale visant l'autocontrainte, en matière de séparation conjugale et de garde d'enfants, tend à se manifester sous la forme d'une incitation à la négociation et, au besoin, à la médiation. Sous le regard de la justice ou des services sociaux, les parties prenantes, dans toutes leurs conduites, sont censées réguler leurs émotions, leurs pulsions et leurs attentes en faisant preuve de bonne foi et en témoignant d'un souci constant des intérêts d'autrui (des enfants et/ou de l'autre parent). Si la plupart jouent le jeu, non sans difficultés ni frictions, certaines savent utiliser le langage du droit et pervertir les valeurs de parité (la coparentalité) pour faire prévaloir leurs seuls désirs et intérêts - qui ne sauraient connaître aucune restriction - et pour dénigrer voire détruire l'autre partie. Mais il convient de contrôler les expressions de violence impulsive pour pouvoir mettre en œuvre la violence insidieuse inhérente à ce détournement des instruments de la négociation et du compromis équilibré. De fait, les avocats prêtent un secours juridique - celui du respect des formes - aux postures les plus unilatéralistes et maximalistes. C'est là toute l'ambiguïté, parfois traumatisante pour les victimes, de la justice.

Postuler le caractère compensateur de cette violence morale - en rapport avec la décomposition de tout le système de pratiques et de valeurs par lequel les femmes adhéraient sur un mode enchanté à leur propre domination (s'auto-dénigrant volontiers), en rapport, autrement dit, avec la décomposition de la violence symbolique (au sens de Pierre Bourdieu) comme garantie de la domination masculine traditionnelle - est un moyen de simplifier les données du problème ${ }^{4}$. Cela ne permet pas seulement d'inscrire ce type de violence dans les changements configurationnels des rapports de genre. Cela permet également de le situer dans l'espace social : il faut certaines ressources spécifiques, lorsqu'on s'adapte superficiellement à la contrainte sociale du dialogue paritaire et du respect réciproque de l'intégrité psychique individuelle pour ne pas avoir à s'adapter réellement à la symétrisation des rapports conjugaux.

En pratique, l'identification de la violence morale conjugale implique souvent d'agir à fronts renversés. Fabienne et les psychologues de l'association livrent ainsi un travail original de catégorisation et de diagnostic qui porte davantage sur la situation que sur l'auteur de violence morale. L'enjeu, à la faveur d'entretiens approfondis sur l'histoire du couple ou de la famille, est de construire des outils intellectuels donnant la possibilité d'attester qu'il y a un auteur et une victime, c'est-à-dire une relation de domination psychologique, plus que de profiler précisément l'auteur (aussi l'étiquette de pervers narcissique - PN - suscite-t-elle de la réserve). L'objectif est de doter les membres de l'association de catégories d'action ajustées à la situation vécue : des catégories d'action privée, salvatrices en termes de positionnement personnel, efficaces en justice. Cela suppose de faire évoluer parallèlement, à travers des interventions médiatiques ou des formations, les catégories de l'action publique - ces schèmes de perception et ces normes d'évaluation, propres aux juges et aux travailleurs sociaux en particulier, axés sur les idées de conflit et de médiation, et par conséquent aveugles à la violence susceptible de se déployer avec leur complicité. Le cœur de mon travail consiste à rendre raison de ces logiques d'action. 


\section{« Égaux à eux-mêmes - mais en pire »}

D'emblée, les pouvoirs publics et les associations ont attiré l'attention sur les risques d'explosion des violences conjugales et des violences sur mineurs en période de confinement. Le jour de l'entrée en vigueur de l'état d'urgence sanitaire, le 23 mars 2020, la présidente de la fédération France Victimes - à laquelle appartient notre association - diffuse une lettre ouverte qui commence par ces mots : «La peur doit changer de camp ${ }^{5}$. » Les dispositifs d'alerte et d'accueil pour les victimes sont promptement mobilisés et mis en avant dans la communication gouvernementale (numéro d'appel 17 ; numéro national de référence 3919, spécialement destiné aux femmes; plate-forme arretonslesviolences.gouv.fr, etc.). Il est annoncé notamment que les ordonnances de protection et d'assistance éducative seront systématiquement prolongées; que les tribunaux continueront à traiter les comparutions ou audiences relatives aux mesures de protection urgentes des victimes de violence intrafamiliale; ou encore qu'il restera possible de saisir en urgence le juge aux affaires familiales - en urgence : c'est-à-dire dans «toute situation mettant en danger l'intégrité physique ou morale d'un enfant, d'un parent ou d'un époux (mauvais traitement à enfants, menaces de mort, violence physique ou psychologique sur conjoint) ».

Dans un bilan rendu public le 9 mai, la fédération France Victimes faisait état du nombre de femmes, victimes relevant de faits dans le couple, accompagnées du 17 mars au 7 mai : sur 11170 d'entre-elles, plus de la moitié (6 108) n'avaient jamais été suivies avant le confinement. Mais comment évaluer - et conceptualiser adéquatement - un «effet» confinement? Je distinguerai, en référence à mon terrain d'enquête, deux cas : celui des victimes accompagnées depuis plus ou moins longtemps et celui des premières prises de contact avec une association.

Sur les 150 membres environ (sur 250 à jour de cotisation) qui bénéficient sur une année des services de l'association, une vingtaine a exigé de la part de Fabienne un investissement de tous les instants. Dès le 21 mars, une membre lui apprend que ses enfants, confiés à l'Aide sociale à l'enfance (ASE), sont retournés sans son avis chez leur «père violent »: «Et moi qui ai subi des violences, je ne verrai plus mes enfants parce que j'ai voulu les protéger. Et ils vont vivre confinés avec leur père violent pendant des semaines, sans aucun contrôle. »

Le $1^{\text {er }}$ avril, je m'entretiens avec Fabienne, qui perçoit en particulier que l'exercice des droits de visite et d'hébergement des parents séparés va cristalliser toute la haine et la mauvaise foi des auteurs. De fait, la possibilité, dans le cadre du décret du 23 mars garantissant cet exercice, de mettre en balance respect de la lettre des jugements et respect de certaines consignes sanitaires ouvre à ces auteurs un «nouveau terrain de jeux ${ }^{6} »$. Dans ce registre, ils n'ont pas déçu : ils se sont accrochés à leurs «droits à », quand les victimes, elles, ont su les relativiser pour mettre au premier plan les intérêts de l'enfant. C'est une Fabienne à la force d'indignation intacte, mais au bord du burn out, que j'interroge le 17 juin : «Les victimes sont les garants de l'accès à l'autre parent. Malgré leurs peurs et les risques que certains pères faisaient courir aux enfants. [...] On n'a pas été surpris outre-mesure. Ce qui m'a déstabilisée, c'est l'ampleur des mails. On le savait très bien. C'est pour ça qu'on avait prévu des séances d'EFT, d'EMDR. Mais c'est quand même perturbant de voir des personnes fonctionner comme ça, pas capables de se remettre en question, alors que tout le monde se remet en question... C'étaient eux les sachants... On ne les change pas : toujours aussi 
menteurs, avides de ce qu'ils n'ont pas, dans cette envie. Ils ont été égaux à eux-mêmes mais en pire. »

Mais le discours et les pratiques de Fabienne ne se coulent dans le moule de l'opposition auteur/victime que pour autant que la situation et le parcours des membres de l'association ont été passés au crible du diagnostic de violence morale. Ce n'est toutefois pas blanc ou noir. Certaines victimes, témoigne Dylan (le seul psychologue homme de l'association), ont été tentées de prendre prétexte des circonstances pour ne pas respecter les jugements. Et rares sont en définitive les auteurs qui les ont bafoués carrément. La différence est plus subtile : on la discerne dans des manières d'être qui, pour les unes, peuvent s'ouvrir - sous des dehors anxieux et agités - à la remise en cause de soi et à la considération de l'intégrité psychique d'autrui ; pour les autres, ne laissent strictement aucun espace à des attitudes de ce type, tout en donnant l'impression inverse. Cette différence, qui creuse pourtant des fossés, n'est visible que par des spécialistes aguerris ${ }^{7}$. Ainsi, explique Fabienne, opposant traits pour traits les victimes et les auteurs, les mêmes circonstances génératrices de stress ont révélé, d'une part, de «l'impuissance », de l'autre, «une incapacité à supporter la frustration ». Elles se sont traduites, d'un côté, par le "respect des jugements et des mesures édictées par le gouvernement», et de l'autre, par un respect de surface, doublé de l'«utilisation de tous les trous possibles et imaginables pour obtenir des prérogatives supplémentaires, imposer des droits qu'ils n'avaient pas, des façons de faire, et, pour ceux qui avaient la garde des enfants, en profiter pour couper les liens avec l'autre parent $\gg$. Il faut bien se rendre compte de quoi il en retourne dans ce dernier cas : il y va, pour le parent dit «auteur», d'une "mainmise totale». Cette «mainmise sur l'enfant ne peut pas ne pas être problématique maintenant qu'on revient à la norme »: «La question que je me pose, vu qu'on nous a seriné l'appel du 119 [numéro national d'urgence pour les enfants en danger], c'est une question par rapport aux enfants : ce fut une période idéale pour les assujettir totalement, leur faire du lavage de cerveau, et les museler. Qui a pu dénoncer ça ? [...] Il y aura beaucoup de leçons à tirer. Ça a montré certaines dimensions qu'on n'avait pas encore explorées. Ça nous a placés face à de la détresse difficile à gérer. Ça a placé les femmes victimes face à une agressivité bien plus importante que la norme [en matière de violence morale] : dénigrement, insultes, mails ont été particulièrement destructeurs ${ }^{8}$. » Une fois encore, c'était attendu. Cela avait été anticipé. Mais cela a traumatisé. Les visages de la violence morale épousent la gamme des dénis de l'objet : en la matière, l'inventivité des auteurs est sans limites et ne laisse pas de déconcerter. C'est un phénomène qui s'inscrit dans la durée et dans la répétition, et qui, en même temps, connaît régulièrement des pics, à certains moments particuliers (fête des pères, anniversaires, Noël, etc.) : «Là, le pic a été immédiat», conclut Fabienne'. L'urgence, le chiffrage instantané des appels et signalements en hausse, la focalisation compréhensible sur la violence physique à l'encontre des enfants, etc., ont peut-être contribué à épaissir le voile entourant la violence morale et les comportements d'étouffement, d'envahissement, par l'auteur, du narcissisme d'autrui au profit de son seul narcissisme ; à faire apparaître, par contraste, toute la difficulté de la preuve ainsi que la limite des instruments de mesure dans les cas d'emprise et de maltraitance psychologique.

\section{Un « effet » confinement ?}

J'ai pu constituer un corpus de 86 messages électroniques - anonymisés - de prise de contact avec l'association reçus entre le 17 mars et le 5 juin 2020. Un aspect de ces premiers messages confirme le constat qui précède à propos de la violence morale : c'est 
l'omniprésence de mots et d'expressions dénotant la durée et la répétition. Tout se passe comme si les personnes concernées étaient en permanence sur le qui-vive, mais aussi prisonnières d'un long processus de dévitalisation, y compris après la séparation.

Quelques exemples : «je me sens affaiblie de jour en jour»; "il continue à être très présent, son ombre est toujours là (exemple : il vient voir si je suis chez moi les soirs où je n'ai pas les enfants) »; «cela fait depuis bien trop longtemps qu'elle se prend pour le marionnettiste »; «Je suis maintenant séparée et divorcée du père de mes enfants depuis 7 ans, mais cela ne suffit pas à faire s'arrêter le harcèlement moral qu'il exerce à mon endroit »; "nous sommes en processus de divorce interminable [...] J'ai pris progressivement conscience de la toxicité de mon mari envers moi et de son emprise sur moi »; «j'ai pu depuis maintenant 8 ans me faire accompagner sur le chemin de l'acceptation que cet homme était un être destructeur»; «je suis sous emprise depuis de longues années »; "je suis épuisée du combat que je mène face à mon conjoint alors que je l'ai quitté il y a plus d'un an et que nous sommes en procédure de divorce. [...] C'est comme si à chaque fois que j'essayais de faire surface, il m'enfonçait à nouveau la tête sous l'eau »; «Il est très jaloux, il vérifie régulièrement mon portable et les chemins que j'ai emprunté. J'ai beaucoup d'exemples. Je suis très fatiguée ».

Sur ces 86 messages, 65 concernent des cas de violence conjugale. Les 21 restants sont en rapport avec d'autres types de violence intrafamiliale (12 cas) ainsi qu'avec des violences exercées par le voisinage (trois cas), par un supérieur hiérarchique (un cas) ou par «l'entourage proche » sans plus de précision (un cas), tandis que, dans quatre cas, la situation de violence ou de harcèlement est dépeinte de manière trop succincte pour qu'il soit possible de discerner sa nature ; les victimes supposées sont toutes des femmes (dans un cas il n'a pas été possible d'identifier le sexe).

Je me concentrerai ici sur les 65 cas de violence conjugale.

Tableau $\mathrm{n}^{\circ} 1$ : messages électroniques reçus par l'association entre le 17 mars et le 5 juin 2020 à propos de cas de violence conjugale

\begin{tabular}{|c|c|c|c|c|}
\hline & Victimes femmes & Victimes hommes & $\begin{array}{c}\text { dont : relatés par } \\
\text { des tiers }\end{array}$ & $\begin{array}{c}\text { dont : évocation du } \\
\text { confinement }\end{array}$ \\
\hline $\mathrm{N}=65$ & 55 & 10 & 9 & 21 \\
\hline $\begin{array}{c}17 \text { mars-10 mai } \\
\mathrm{n}=37\end{array}$ & 31 & 6 & 6 & 14 \\
\hline $\begin{array}{c}11 \text { mai-5 juin } \\
\mathrm{n}=28\end{array}$ & 24 & 4 & 3 & 7 \\
\hline
\end{tabular}

Au total, moins du tiers des messages reçus du premier jour du confinement (17 mars) au début de la phase 2 du déconfinement ( 5 juin) font référence à ces circonstances inédites (21 sur 65) ; et encore ne s'agit-il, dans quatre cas, que d'allusions impersonnelles, comme en passant. Pendant la période du confinement proprement dit (17 mars-10 mai), la proportion est plus importante (14 sur 37$)$. Il est à noter que la moitié des messages d'hommes ou à propos d'hommes évoquent le confinement (5 sur 10). Il en va de même de quatre messages sur neuf écrits par des tiers. Le seul message qui établit explicitement une corrélation directe entre l'événement et la décision de tirer les conséquences de la violence morale subie date du 27 mai : "Le confinement avec le covid que j'ai eu ont été un électrochoc qui m'ont donné la force de prendre la décision de me séparer. Depuis que je lui ai annoncé, vendredi dernier, il essaie de me manipuler. » Un deuxième (10 mai) est plus allusif : «Bonjour, je subis une pression constante et psychologique de la part de mon conjoint et père de ma fille de 12 mois. 
J'ai enfin pris conscience. J'aimerais avoir votre aide pour sortir de cette situation svp. Je $m$ 'appelle $X$. Je suis en confinement avec lui. »

Le cas de figure qui domine ( 8 messages) est le suivant : le confinement intervient alors que des séparations sont en cours, que des procédures de divorce ont été engagées, que des actes forts ont été posés (par exemple, un signalement pour violence psychologique). Ces messages sont autant d'appels au secours. Il importe de gérer soit un départ précipité, soit une cohabitation forcée et particulièrement éprouvante. Pour un père qui souhaitait un divorce à l'amiable et obtenir la garde alternée, tout devient ainsi plus compliqué : "Dans le contexte $d u$ confinement, il est donc évident que le quotidien est bien plus dur car pas de soupapes de décompression dans la journée. Toutes mes initiatives pour apaiser les tensions et proposer des solutions sont perçues comme incomplètes, manipulatrices. »

Très proche de cette configuration, on a celle-ci (3 messages) : le confinement active ou réactive une décision de séparation ou de divorce qui était latente, ou qui avait déjà été envisagée peu de temps auparavant et repoussée : «Depuis le début du confinement, la situation s'empire et devient insupportable et $j$ 'ai reparlé à $X$ de mon intention de séparation. $J$ Je vois que $X$ cherche à manipuler notre entourage en me faisant passer pour le responsable de tout ça. Elle me dit que c'est moi qui suis manipulateur. »

On note aussi deux demandes de conseil juridique liées au confinement. Enfin, deux derniers messages se distinguent par le fait qu'ils ne revêtent aucun caractère d'urgence. Ici, le confinement semble jouer comme période favorisant la reviviscence de traumatismes passés, ou propice à la cogitation et au retour sur soi, suspension des activités ordinaires aidant. Une femme, probablement jeune, remarque dans un long message que «cette période de confinement nous a enfermés avec nos souffrances et peurs non élucidées que la vie active avait tenté de maquiller jusque-là ». Libérée depuis un an d'une relation qui l'a beaucoup faite souffrir, elle ne cesse de s'interroger et cherche à «débloquer» sa vision. Après s'être abondamment renseignée sur internet pendant le confinement, elle voudrait simplement pouvoir obtenir «confirmation que j'avais à faire à un manipulateur pervers (narcissique je pense aussi mais je ne suis pas experte) ». Une jeune trentenaire, conseillée par une amie psychologue, s'adresse également à l'association: "Je ne suis pas dans une situation d'urgence mais je ressens le besoin de commencer à en parler enfin. » Elle a vécu en couple pendant dix ans avec «une mauvaise personne qui [l]'a énormément manipulée ». Séparée depuis deux ans, elle voit maintenant ressurgir, dans le contexte du confinement, des sensations refoulées: "J'ai réussi à partir il y aura bientôt 2 ans [...]. Mais qui dit confinement dit réflexion lol et mes blessures non guéries remontent. »

Ces deux exemples sont apparemment anecdotiques. Ils ne sont pas d'une gravité qui appelle un suivi prioritaire de l'association (les personnes concernées ne sont pas mariées et n'ont pas d'enfants) ${ }^{10}$. Ils n'en témoignent pas moins très bien de la nature profondément envahissante et destructrice, pour l'intégrité psychique, des comportements qui réduisent systématiquement l'autre au statut de pur objet sans qualités propres.

Sur les 65 messages étudiés, les mots qui reviennent le plus souvent pour qualifier ces mécanismes interactifs sont :

- violence(s) (44 occurrences), psychologique(s) (10), conjugale(s) (9), morale(s) (9), physique(s) (5), verbale(s) (5), et violent(s) (11 occurrences), en rapport à «des 
actes », «des reproches », ou pour désigner des agents (« un père », «mari », «deux ex», «une mère », « un homme »);

- harcèlement (20 occurrences), surtout moral (10), le verbe harceler étant en outre utilisé à deux reprises et une personne étant désignée comme «harcelante »;

- manipulation(s) (12 occurrences), à quoi s'ajoutent onze emplois du verbe manipuler et six occurrences de manipulateur sous forme substantive (3) ou adjectivale (3) ;

- emprise (12 occurrences).

S'agissant de caractériser la personne même, c'est le substantif pervers narcissique qui vient en tête avec 17 occurrences, comprenant sept occurrences de l'acronyme PN (dans sept messages différents de femmes) et trois occurrences du féminin perverse narcissique (dans deux messages d'hommes).

Il est possible que, pour les victimes de violence morale qui se pensaient plus ou moins tirées d'affaire avant le confinement, l'avènement de cette période - synonyme de relâchement brutal du rythme ordinaire des activités sociales, porteuse, en même temps, d'une menace impalpable, «sans visage ni règles ${ }^{11}$ », mortifère - ait fait écho à des intériorités psychiques marquées par le sentiment d'une menace imminente, c'est-à-dire à des vécus d'emprise. De là, le besoin de fixer enfin une vigilance sans objet, de régler des problèmes trop longtemps gardés sous le boisseau.

Retenons toutefois - aussi réduit soit l'effectif considéré - que plus des deux tiers des messages analysés dans cet essai ne font pas la moindre allusion au confinement. Rien, hormis leur date, ne permet de deviner qu'ils ont été envoyés durant cette période : comme si, pour toutes ces personnes en demande d'aide, celle-ci n'avait été qu'une simple péripétie par contraste avec le confinement de leur psychisme dans les forteresses de contrôle, de dénigrement et de domination érigées par les auteurs de violence.

On aimerait croire que les pouvoirs publics et les personnels concernés - justice, travail social, etc. - puissent un jour prendre pleinement conscience des caractéristiques de ce phénomène si déstabilisant et dérangeant. Cela passe, assurément, par une amélioration des formations sur les thématiques psychologiques de l'emprise, du déni ou de la manipulation. Mais cela suppose aussi d'appréhender le problème sociologiquement, en montrant par exemple comment, depuis le début des années 1980, le creusement observable des inégalités de richesse entre hommes et femmes et le développement d'une violence conjugale «invisible», morale ou psychologique, fondée sur le déni actif de l'intégrité psychique de l'Autre (tout se passant comme si le «despote altruiste » cher à Gary Becker avait fait place à un despote égotique, faussement altruiste), sont les deux faces d'une même entreprise visant à rétablir une domination masculine absolue, aussi bien à l'encontre du droit (qui, à la lettre, ne lui assure plus aucun fondement, et dont il convient donc de se jouer) que des dynamiques sociales et politiques de symétrisation des rapports de genre.

\footnotetext{
1 Le sigle EFT («Emotional Freedom Techniques») désigne un ensemble de techniques de libération émotionnelle inspirées de l'acupuncture et de la psychothérapie comportementale. L’EMDR (« Eye Movement Desensitization and Reprocessing ») est une thérapie comportementale reposant sur les mouvements oculaires et
} 
favorisant en particulier le rappel des traumatismes. Elle est de plus en plus utilisée dans le traitement des états de stress post-traumatique.

${ }^{2}$ Paul-Claude Racamier, «Folie familiale, délire et catastrophe », Groupal, n 2, 1996, p. 25.

${ }^{3}$ Dans cette perspective, la notion d'informalisation, construite par Cas Wouters, a été cruciale pour affiner la théorie éliasienne. Voir Cas Wouters, Michael Dunning, Civilisation and Informalisation. Connecting LongTerm Social and Psychic Processes, Basingstoke, Palgrave Macmillan, 2019.

${ }^{4}$ Mon propos n'est évidemment pas de dire que «la violence morale » aurait pris purement et simplement la place de «la violence symbolique ». Les formes de cette dernière - entendue comme phénomène universel sont probablement plus éclatées et instables. Ma réflexion, à ce sujet, en est encore à un stade préliminaire. Voir Marc Joly, «Le genre des capitaux », https://journals.openedition.org/lectures/42127. Il s'agit d'une longue note critique consacrée au livre de Céline Bessière et Sybille Gollac, Le Genre du capital. Comment la famille reproduit les inégalités, Paris, La Découverte, 2020. La lecture de ce livre m'a aidé à avancer dans l'approfondissement de ma problématique.

${ }^{5}$ https://www.france-victimes.fr/index.php/presse/communiques-de-presse

${ }^{6}$ Selon l'expression de Dylan, psychologue à l'association (entretien du 24 juillet 2020).

${ }^{7}$ D'après le code civil, les juges sont censés pouvoir apprécier, lorsqu'ils se prononcent sur les modalités d'exercice de l'autorité parentale, aussi bien "l'aptitude de chacun des parents à assumer ses devoirs et respecter les droits de l'autre» que "les pressions ou violences, à caractère physique ou psychologique, exercées par l'un des parents sur la personne de l'autre». Dans les faits, surtout quand les pressions ou violences sont purement d'ordre psychologique, beaucoup paraissent toutefois mal armés et sont facilement induits en erreur.

${ }^{8}$ Entretien avec Fabienne (17 juin 2020).

${ }^{9}$ Le cas (minoritaire) des membres de l'association confinées avec des auteurs a été ici laissé de côté. Disons seulement que la violence est restée morale : «Pas de coups, pas de bleus - elles sont restées » (entretien avec Fabienne, 17 juin 2020).

${ }^{10}$ De manière générale, il est certain que la majorité des messages n'a pas donné lieu à adhésion.

${ }^{11}$ Selon les mots de Dylan (entretien du 24 juillet 2020), et pour reprendre une hypothèse issue de sa clinique avec des personnes dépressives et des femmes anciennement violentées. 